\title{
A PMD-adaptive DBP Receiver Based on SNR Optimization
}

\author{
Gabriele Liga $^{1}$, Cristian B. Czegledi ${ }^{2}$, and Polina Bayvel ${ }^{1}$ \\ 1. Optical Networks Group, Dept. of Electronic \& Electrical Engineering, University College London, London WC1E 7JE, UK \\ 2. Dept. of Electrical Engineering, Chalmers University of Technology, Gothenburg SE-412 96, Sweden \\ gabriele.liga.11@ucl.ac.uk
}

\begin{abstract}
We propose a novel adaptive digital backpropagation (DBP) scheme that tracks the fiber polarization-mode dispersion via the optimization of the signal-to-noise ratio. Gains of up to $1.4 \mathrm{~dB}$ over conventional DBP are achieved.
\end{abstract}

OCIS codes: (060.4510) Optical communications; (060.1660) Coherent communications.

\section{Introduction}

Digital backpropagation (DBP) is today the most widespread digital technique to undo signal distortions due to fiber nonlinearity. Ideally, when DBP is applied to the entire transmitted wavelength-division multiplexing (WDM) bandwidth, all nonlinear effects generated by signal-signal interactions should be fully compensated. However, many non-ideal conditions can interfere with this process. One of them is, notably, the fiber polarization-mode dispersion (PMD), which is not compensated for in the most conventional implementation of DBP [1,2]. The reason for that lies in the fact that the DBP would require the information on how PMD evolves along the link (possibly at each DBP step), and this information is typically unavailable at the receiver. Previous works [3-5] have attempted to somehow produce estimates on the fiber PMD sections in order to improve the performance of DBP. These attempts of emulating the PMD distribution along the fiber in the backward propagation resulted in a significant signal-to-noise ratio (SNR) gain (e.g. $1.1 \mathrm{~dB}$ of average gain in [4]). However, these works only tackled relatively small transmitted and backpropagated bandwidths, for which the detrimental effect of PMD on DBP can be marginal for the moderate PMD values of modern fibers $(\leq 0.1$ $\mathrm{ps} / \sqrt{\mathrm{km}})$. For multichannel DBP, PMD is still a fundamental obstacle that prevents from attaining the full nonlinearity compensation gain $[2,6]$. This detrimental effect can be observed in Fig. 1, where the SNR is plotted as a function of the transmitted power for different DBP bandwidths (system parameters described in Table 1). In this paper, we study a novel adaptive DBP scheme that effectively counteracts the limitations imposed by PMD on wide-band nonlinearity compensation. The proposed scheme acquires knowledge on the fiber PMD sections through the optimization of the SNR after DBP is applied.

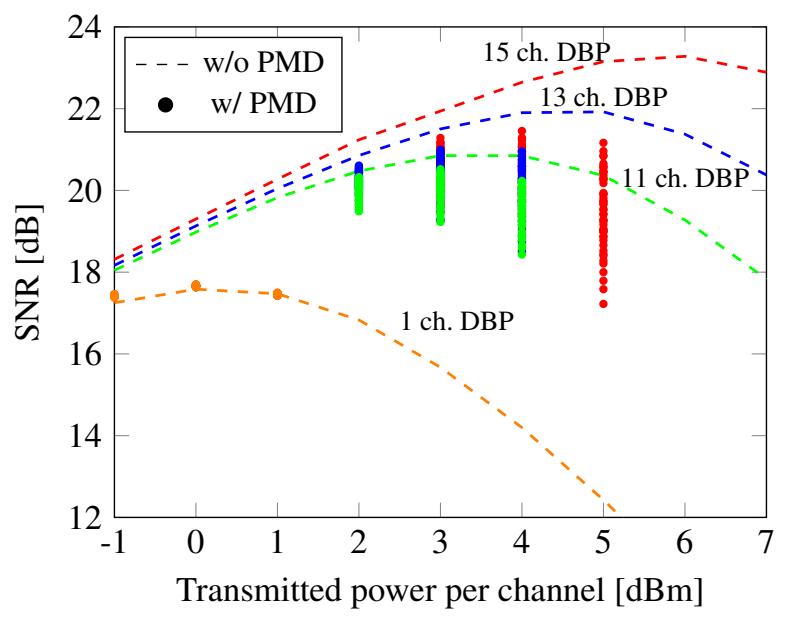

Fig. 1: SNR vs. transmitted power for various DBP bandwidths without PMD and with PMD using a conventional DBP scheme. System parameters in Table 1.

\begin{tabular}{cc}
\hline System Parameters & Value \\
\hline Symbol rate & $32 \mathrm{GBaud}$ \\
No. of channels & 15 \\
Channel spacing & $33 \mathrm{GHz}$ \\
Mod. format & PM-16QAM \\
Span length & $100 \mathrm{~km}$ \\
No. of spans & 10 \\
Fiber type & $\mathrm{SSMF}$ \\
PMD & $0.05 \mathrm{ps} / \sqrt{\mathrm{km}}$ \\
Fibre correlation length & $100 \mathrm{~m}$ \\
EDFA noise figure & $4.5 \mathrm{~dB}$ \\
\hline Simulation Parameters & Value \\
\hline Numerical method & Log-step split-step Fourier \\
Oversampling ratio & 2 \\
DBP steps & Up to $700 / \mathrm{span}$, log-step \\
\hline
\end{tabular}

Table 1: Parameters of the system investigated in this work. 


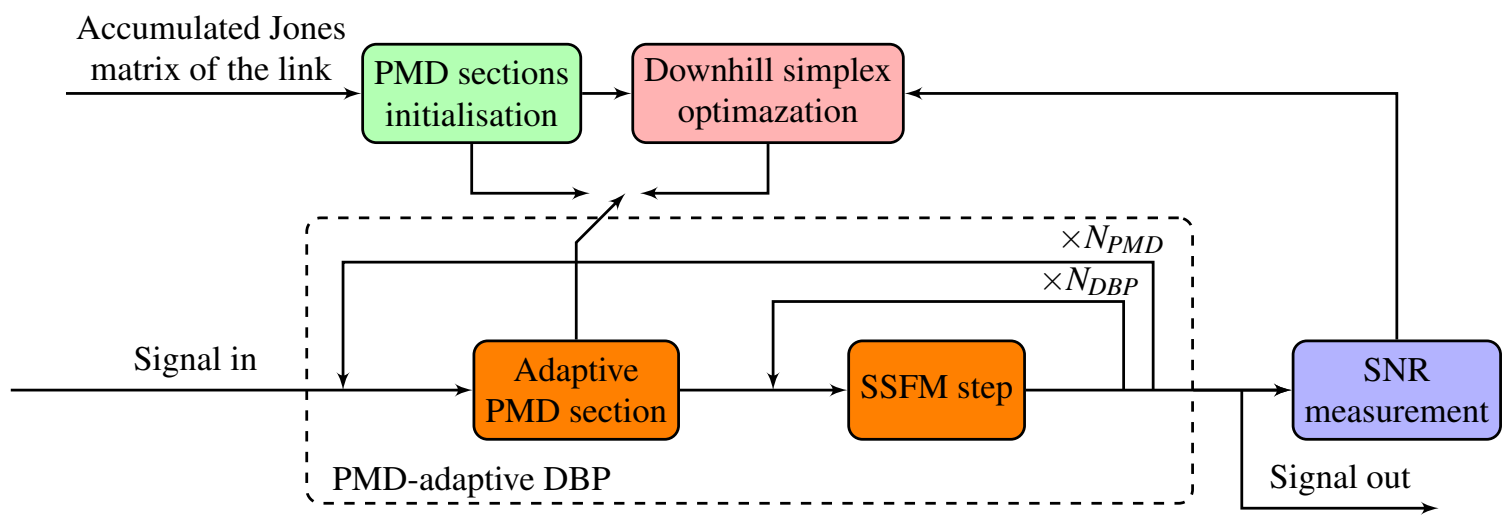

Fig. 2: Schematic diagram of the adaptive DBP algorithm used in this work (SSFM: split-step Fourier method).

\section{Proposed Adaptive Scheme}

The approach proposed in this paper aims to optimize the performance of modified DBP schemes implementing reverse PMD steps as in [3-5]. An optimization of the PMD sections was also proposed in [3] to obtain solutions that match the accumulated Jones matrix of the link. The set of PMD sections that are solutions to such an optimization problem are infinitely many and, in general, having sections does not guarantee the convergence to the true fiber solution, which would guarantee optimal performance. Moreover, as the transmitted bandwidth is increased, it obtaining PMD sections that are close to the ones of the fiber is less likely. In order to overcome this problem, the method here investigated uses directly the SNR after DBP as the objective function to maximize. Despite the high complexity required in the calculation of the objective function, no additional complexity is required for systems already implementing DBP in real-time, except for the calculation of the SNR. A schematic diagram of the proposed PMD-adaptive DBP scheme is illustrated in Fig. 2. The DBP block implements a PMD section each $N_{D B P}$ DBP steps to compensate for the accumulated PMD. Each PMD section consists of the product of two matrices: a constant polarization rotation and a diagonal frequencydependent matrix describing the differential group delay of the section. Here, the PMD sections are inserted once every fiber span. The adaptation of the PMD sections is only based on the knowledge of the accumulated Jones matrix of the link. Since no lumped polarization demultiplexing stage is adopted in the diagram in Fig. 2., an effective bootstrap procedure for the PMD sections is required. To this aim we adopt the algorithm in [3], which, as discussed in Sec. 1, returns a combination of PMD sections that effectively equalize the Jones matrix of the link. This distribution is fed into the DBP block and into the actual optimization algorithm for the PMD sections which is a variant of the so-called downhill simplex algorithm [7]. The algorithm searches for the optimum SNR as a function of the $N_{P M D}$ PMD sections, each represented by a set of 3 Stokes angles and 1 DGD value. The downhill simplex algorithm is a derivative-free method for nonlinear programming problems that only requires a single SNR evaluation per iteration. This makes it particularly suitable for a computationally-intense optimization such as the one performed in this paper.

\section{Results and Discussion}

The performance of the proposed scheme are investigated for the system described in Table 1. DBP is operated over 3 different bandwidths (BWs): 11, 13 and 15 channels. For each DBP BW the transmitted power is changed according to Fig. 1 to achieve the optimal SNR. Different PMD realizations are also generated for each BW. The obtained results are shown in Fig. 3. Fig. 3(a) shows the SNR evolution at each algorithm iteration for the 3 DBP BWs investigated. The optimization algorithm is stopped after 2000 iterations. It can be observed that the initial value of the SNR changes according to the initial PMD section arrangement provided by the initialization algorithm. This depends on the specific PMD realization and solution provided by the algorithm. In this particular instance, the starting SNR for a DBP BW of 11 channels is $0.5 \mathrm{~dB}$ higher than the one for a DBP BW of 13 channels. However, as expected, the 13 channel-DBP converges to a higher SNR than the 11 channel-DBP one ( $21.0 \mathrm{~dB}$ and $20.5 \mathrm{~dB}$, respectively). For the 15 channel-DBP case, 3 different PMD realizations are shown. It can be seen that the SNR is not entirely converged after 2000 iterations, although, from these preliminary results, it could be argued that the steady-state SNR does not depend on the initial PMD sections' arrangement. This would suggest a convexity of the objective function. More PMD realizations are, however, required to confirm this behavior. Smaller DBP BWs (e.g. 11 channel-DBP) can be observed to converge faster than larger ones (e.g. 15 channel-DBP). This is explained due to the different gaps between the starting SNR and an ideal 


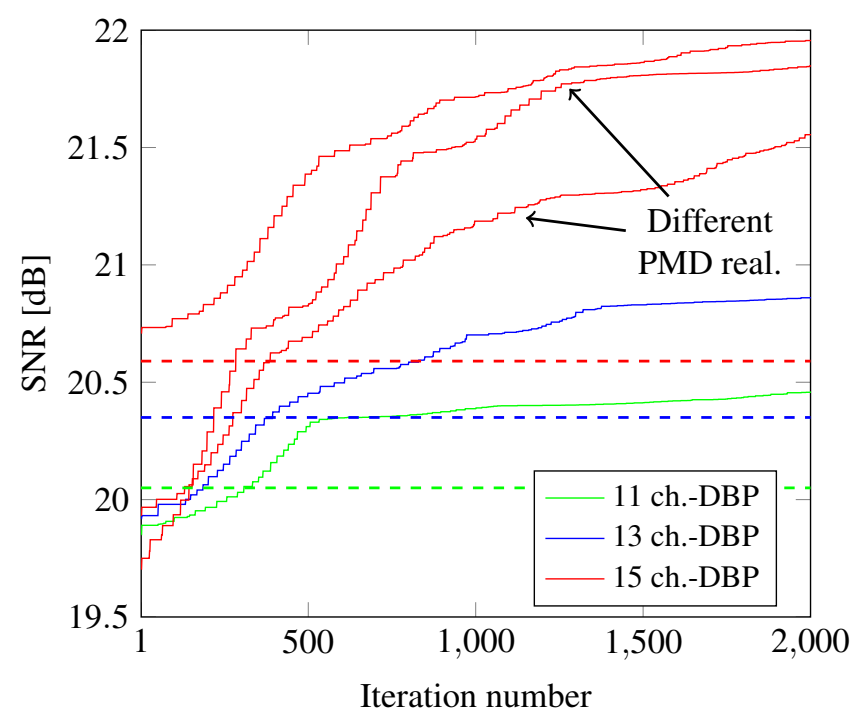

(a)

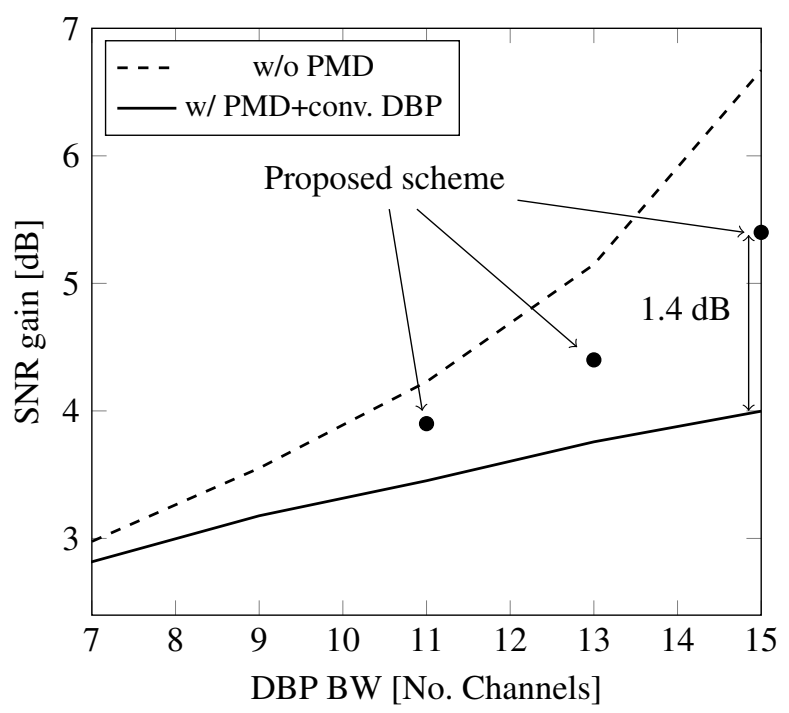

(b)

Fig. 3: (a) Convergence of the SNR for different DBP BWs. Dashed lines indicate the average SNR for conventional DBP. (b) SNR gain over dispersion compensation-only vs. DBP BW w/o PMD, and w/ PMD using conventional (average SNR gain over 50 PMD realizations) and PMD-adaptive DBP.

nonlinearity compensation SNR (no PMD), as shown in Fig. 3(b). Fig. 3(b) shows the DBP SNR gain over a dispersion compensation-only receiver as a function of the DBP BW, and for 3 different cases: no PMD in the fiber; with PMD and a conventional DBP scheme; with PMD and using our proposed PMD-adaptive scheme. The gap between the SNR gains achievable using a conventional DBP scheme with and without PMD increases with the DBP BW. This indicates a larger potential gain for the proposed optimization algorithm which, ideally, is expected to converge to SNRs achievable in the absence of PMD. Our proposed scheme achieves (using 2000 iterations) SNR gains of up to $0.4 \mathrm{~dB}, 1.0 \mathrm{~dB}$ and $1.4 \mathrm{~dB}$ for 11 channel-, 13 channel- and 15 channel-DBP, respectively, compared to conventional DBP. The residual gap to the ideal nonlinearity compensation gains is attributed to the reduced number of iterations performed and to the use of only 10 PMD sections.

\section{Conclusions}

We investigated, for the first time, the performance of an adaptive DBP scheme that learns the evolution of the fiber PMD through the optimization of the SNR at the DBP output. This approach effectively counteracts the detrimental effect of PMD on wide-band nonlinearity compensation. Within 2000 iterations, the proposed approach achieves SNR gains of up to $1.4 \mathrm{~dB}$ compared to the conventional DBP implementation. Further study on potential singularities of the algorithm are left for future work.

\section{Acknoledgements}

The UK EPSRC Programme Grants UNLOC EP/J017582/1 and INSIGHT EP/J017582/1 are gratefully acknowledged for the financial support.

\section{References}

[1] E. Ip, "Nonlinear Compensation Using Backpropagation for Polarization-Multiplexed Transmission,” J. Lightwave Tech.20 939-951 (2010).

[2] G. Gao, X. Chen and W. Shieh, "Influence of PMD on fiber nonlinearity compensation using digital back propagation," Opt. Express 20 1440614418 (2012).

[3] C. B. Czegledi, G. Liga, D. Lavery, M. Karlsson, E. Agrell, S. J. Savory, P. Bayvel, "Polarization-Mode Dispersion Aware Digital Backpropagation Cristian," in Proc. European Conference on Optical Communication (2016).

[4] C. B. Czegledi, G. Liga, D. Lavery, M. Karlsson, E. Agrell, S. J. Savory, P. Bayvel, "Digital backpropagation accounting for polarization-mode dispersion," Opt. Express 25 1903-1915 (2017).

[5] K. Goroshko, H. Louchet, and A. Richter, "Prediction of Accumulated DGD Evolution for PMD-Resistant Digital Back Propagation," in Proc. International Conference on Transparent Networks (2017).

[6] G. Liga, C. B. Czegledi, E. Agrell, R. K. Killey, P. Bayvel "Ultra wideband nonlinearity compensation in the presence of PMD," in Proc. European Conference on Optical Communication (2016).

[7] A. Nelder, H. Mead “A simplex method for function minimization,” Computer Journal. 7 308-313 (1965). 